\title{
Image Forgery Detection by using Machine Learning
}

\author{
J.Malathi, B.Narasimha Swamy, Ramgopal Musunuri
}

\begin{abstract}
Dense local descriptors and AI havebeen utilized with achievement in a couple of employments, as classificationof surfaces, steganalysis, and bowing zone. We build up a newimage counterfeit marker creating unequivocal descriptors recentlyproposed in the steganalysis field reasonably joining some of suchdescriptors, and redesigning a SVM classifier on the availabletraining set. The issue with the present making is that majorityof them see certain highlights in pictures changed by a particular tamperingmethod, (for example, duplicate move, joining, and so forth). This proposes the structure does notwork always transversely over different evolving frameworks. Mix of no under two pictures to make a completely phony picture is known as Image structure. It winds up being difficult to disengage between certified picture and phony picture in light of the closeness of different astounding changing programming endeavors. In this paper, we propose a two phase imperative altering way to deal with oversee direct learn featuresin referencing to see changed pictures in various pictureformats.
\end{abstract}

\section{INTRODUCTION}

Inthe modernized time, there are a tremendous volume of arranged pictures by techniques for online systems association media platformssuch as Facebook or Flickr. The undertaking of controlled pictures can be sharedvery attainably and can be used to mislead watchers from this present reality. This may result in veryserious results so the validness of cutting edge pictures is truly required.Image Forgery or modification of digital images is not a new concept. It is as old as Photography. But due tothe fast development of technology, In Today's time we cannot imagine the exact usage of digital imagesevery day for various purposes [1].It is said that, - an image tells a thousand words. Images are used toexplain tough concepts, and inspire us easily in each and every field. With the easily availability of internet,digital cameras and editing software's it is very easy to create a fake image without any training or extraknowledge. The trend of modification in digital images is increasing day by day. In various cases, where theimages are used as evidences, the authenticity of images is important to prove in that cases then only theimages can be used as a proof. Digital image forgery or we can say that tampering of digital images havebecome one of the major problems in crimes. There are many ways through which the image can be modified. Combining all those ways the three waysare image retouching forgery, Spliced picture corrupted, copy move duplicate [2]. Picture joining is one of the wayof changing an image that copies to some degree an

Revised Manuscript Received on April 12, 2019. India.

J.Malathi, Sri C.R.Reddy College of Engineering College, Eluru.A.P,

B.NarasimhaSwamy, PVP Sidhhartha Institute of Technology, Vijayawada,A.P, India.

RamgopalMusunuri, PVP Sidhhartha Institute of Technology, VijayawadaA.P, India. outstanding picture and paste it onto another image to make afake picture, and it is dominatingly trailed by post directing structures, for instance, neighborhood/everything thought about obscuring, compression, and resizing [3].It is generally called picture procedure. Composite picture is an image made by thecombination of something near two than two pictures and is joined to shape a specific picture.There are in a general sense five sorts of picture criminological contraptions techniques. Pixel Based Techniquesconsists of that contraptions which helps in watching courses of action from the standard all around quantifiable that are to be appeared at thepixel level. Connection based Techniques join those contraptions which helps in acknowledgment the certain correlationsintroduced by a predefined lossy weight structure.

This social gathering has been filling in starting late on the forgerydetection issue, focusing on systems subject to camerasensor battle, a.k.a. PRNU (photograph reaction nonuniformity)noise [1], [2], [3], [4] and on frameworks subject to thick localdescriptors and AI [5]. As such, we decidedto scan for after both these frameworks for locale, on two separatelines of advancement, with the brightening behind mixing choices at somelater time of the structure. Indisputably, it is striking [6] that,given the undeniable sorts of defilement experienced in practice, and the wide receptiveness of colossal photograph changing tools,several affirmation approachs ought to be utilized at the relative timeand sensibly joined to get the best possible performance. In light of this thought, we in like course inspected for after a third line of improvement dealing with a structure for copy move forgery space which, yet material just to a fraction of the picture set, gives absolutely solid outcomes.

Joined picture creation solicitation ought to be conceivable through various ways. Among those ways, light assortments from the standard are useful for joining affirmation. Two systems for Illumination based fake Detection are Geometry based structures and shading based methods. Geometry based frameworks helps in watching eroticisms in zone of light source between Particular articles and contraptions. Shading based frameworks helps in watching abnormalities in the correspondence between article shading and light shading [2]. In this paper we used shading IL luminance for trickiness introduction. In each logic manual joint effort is must. Nowadays it is difficult to trust in pictures. A human eye can't separate between the genuine picture and fake picture. So we impact self-loader to duplicate structure for the space of joined pictures that makes utilization of AI classifiers where the decision is taken byclassifier.

Published By:

Blue Eyes Intelligence Engineering 


\section{DENSE LOCAL DESCRIPTORS FOR SPLICING DETECTION}

Several structures have been proposed in the last decade for joining attestation subject to AI. Major efforts have been given to find remarkable exact models for natural images in order to single out the features that guarantee the highest discriminative power. As continually as could be standard reasoning about the present situation, to get moremeaningful estimations, change space features have beenused, as in [7] where the image encounters square watchful discrete cosine change (DCT) with various square sizes and firstorder(histogram based) and higher-demand (advance probabilities)features are amassed and joined together. Given the amazing resultsobtained to the degree demand exactness, an exhaustive Markovbasedscheme in DCT and DWT spaces is followed in [8].

Sporadic, the system proposed [7] was invigorated by prior work finished in steg analysis which, paying little regard to the obviousdifferences concerning the drive apparent confirmation field, pursues a all around unclear to objective, that is, seeing verifiably invisible alterations of the standard attributes of an image.The same way is followed in the bowing assertion techniqueproposed in [9], in setting on a structure proposed for steg analysis in [10]. The essential duty wires into deriving the features reliant on some co-occasion structures computed on the clarification behind limitation need bungle picture (in addition called residual image). Beyond question, showing the residuals as opposed to the pixelvalues is unfathomably sensible in these low-level procedures (not basedon picture semantic), since the image content does not helpdetecting neighborhood changes and should be affirmed unmitigated.

Concerning fake introduction, unequivocally, consideringthat joining routinely shows sharp edges, it is reasonableto portray quantifiably some edge picture, which can alsobe the yield of a certain high-pass channel (like a right hand of first demand). As a further upheld position, the holding up picture has amuch littler amazing degree than the first, allowingfor a limited and solid quantifiable framework by methods ofco-occasions.

The organizing course depicted out above, start at now proposed in[10], can be accordingly plot in the running with advances

1) estimation of the high-pass residuals;2) truncation and quantization;3) incorporate extraction subject to co-occasion cross areas ofselected neighbors;4) structure of a sensible classifier on the framework set.Given its actuating procedure concerning considering, and some supporting resultsobtained in the connection, we will search for after in this way, here.Nonetheless, unfathomable choices must be made,beginning from the high-pass channel, to complete with the classifier,which impact unequivocally on the execution and require lengthydevelopment and testing. Fortunately, we can rely upon theprecious results portrayed in a perpetual work on steg analysis where colossal have been consideredand pushing down, and made open online to the examination sort out. Specifically, in different clear high passfilters have been considered, both straight and nonlinear,with evident help, accumulated quantization and truncation strategies for the stores have been executed and, based on some starter tests, the usage of some selected

groups of neighbors for co-occasion figuring has been suggested.

\section{THE PROPOSED WORK\& RESULTS}

The techniques used in the proposed forgery detection method are explained in this section. System Framework is shown in Fig1.

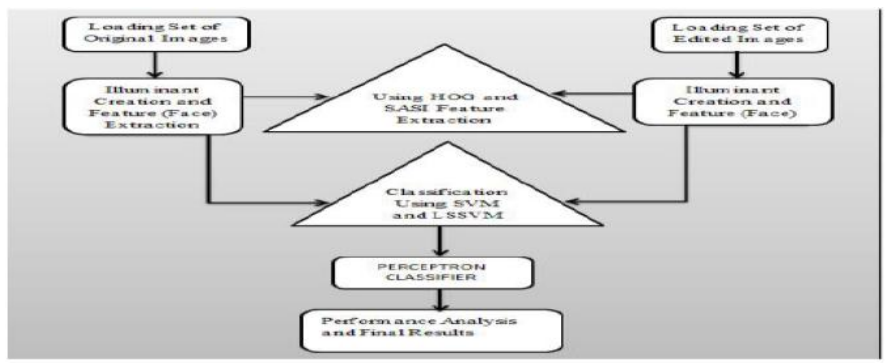

Fig 1: System Framework

Overview of System framework: In this paper, we used the concept illuminant color inconsistency and machine learning classifiers for forgery detection. The method mainly consists of the following steps.

A Estimation of illuminant color: Illuminant color is estimated for input images. Then new images are created i.e. illuminant map for each image that is read.

B Extraction of face: All faces present in one image and corresponding all faces of other individual images are extracted for investigation. Result of Face extraction is shown in Fig 2

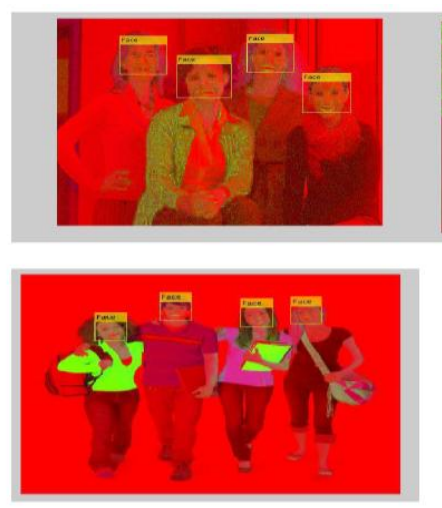

Fig 2: Results of color illuminance, Feature extraction, and Face extraction of various edited images usingSVM and LSSVM

C Extraction of highlights: There is a need to clear textural and inclination highlights. The data in regards to spatial approach of shading in a picture is given by Image surface. This effects us to acknowledge substance of picture completely. Social affair, SASI, highlights are isolated.

D HOG: Histogram of Oriented Gradients: We utilized HOG that is an edge descriptor and is utilized with the genuine focal point of thing exposure in PC vision and picture overseeing. Histogram of Oriented Gradient descriptors or edge headings essentially portrays the shape 
and closeness of neighborhood object inside a picture. The picture is detached into little zones called cells with the credible goal of highlight extraction. Social event introduction for the pixels inside the phone are figured. Highlight descriptor is the blend of these histograms. There might be designs in the extracted HOG features depending on the size and shape of face under construction. So feature vectors of fixed length should be obtained [9].

\section{CONCLUSION}

The everything considered work is executed by the usage of different AI classifiers SVM. The upside of proposed work is that customer affiliation is completely depleted and is set in the attitude for seeing a wide dimension of made pictures. In this work, an improved forgery detection framework .This work is basically fit for seeing joined pictures. As future work, we will join other picture changes, for instance, DCT as the base intertwine information. We will in like way look at if other crucial learning structures such as Deep Belief Networks will improve the execution of feature learning. In like way, wewill continue with our undertakings to physically stamp more ground feelings from various datasets suchas the Columbia Image Splicing dataset [15] as it likewise joins BMP record plans. This will draw in the essential understudy to modify more qualities of changed zones and ensurebetter exactness for balanced region control transversely over different image file formats.

\section{REFERENCES}

1. Christian Riess and Tiago Jose de Carvalho,"Exposing Digital Image Forgeries by Illumination Color Classification", IEEE Transactions OnInformation Forensics And Security, vol. 8,2013

2. Arunvinodh $\mathrm{C}$ and M.F, Reshma P.D, "Image Forgery Detection Using Svm Classifier," IEEE Sponsored 2nd International Conference onInnovations in Information Embedded and Communication Systems", 2015.

3. Bo Xu, Guangjie Liu, and Yuewei Dai , "Detecting Image Splicing Using Merged Features in Chroma Spacell, the Scientific WorldJournal",2014.

4. Luo, Weiqi, Jiwu Huang, and GuopingQiu, "Robust detection of region-duplication forgery in digital imagel, Pattern Recognition", ICPR18th International Conference on. Vol. 4.IEEE, 2006.

5. B. L. Shiva Kumar and Lt. Dr.Santosh, "Detecting Copy Move Forgery in Digital Images: A Survey and analysis of current methods", GlobalJournal of Computer Science and Technology, vol.10, 2010

6. M. K. Johnson and H. Farid,"Detecting photographic composites of people", in Proc. 6th Int. Workshop on Digital Watermarking, Guangzhou,China, 2007.

7. P. K. Bora and SandeepGholap, "Illuminant Colour Based Image Forensics", Proc. IEEE Region 10 Conf, pp. 1-5, 2008.

8. S.L.Jothilakshmi and V.G.Ranjith,"Automatic Machine Learning Forgery Detection Based On SVM Classifier", (IJCSIT) InternationalJournal of Computer Science and Information Technologies, Vol. 5 (3), 2014, 3384-3388, 2014.

9. J.A.K. Suykens J. De Bra banter, L. Lukas and J. Vandewalle,"Weighted least squares support vector machines: robustness and sparseapproximation", Elsevier, Neurocomputing 48 85-105, 2002.

10. D. Zou, Y.Q. Shi, W. Su, and G.R. Xuan, "Steganalysis based on markovmodel of tresholded prediction-error image," International Conferenceon Multimedia and Expo, pp. 13651368, 2006. 